\title{
Possibility of Quasi-Steady-State Operation of Low-Temperature LHD-Type Deuterium-Deuterium (DD) Reactor Using Impurity Hole Phenomena - DD Reactor Controlled by Solid Boron Pellets - ${ }^{*}$
}

\author{
Tsuguhiro WATANABE ${ }^{1, * *)}$, Osamu MITARAI ${ }^{2)}$ and Hideaki MATSUURA ${ }^{3)}$ \\ 1) National Institute for Fusion Science, 322-6 Oroshicho, Toki 509-5292, Japan \\ ${ }^{2)}$ Tokai University, 9-1-1 Toroku, Higashi-ku, Kumamoto 862-8652, Japan \\ ${ }^{3)}$ Kyushu University, 833 Motooka, Nishi-ku, Fukuoka 819-0395, Japan
}

(Received 15 November 2014 / Accepted 1 April 2015)

\begin{abstract}
The burn control of deuterium-boron fusion reactor (DB reactor), in which the deuterium is primary fuel and the boron is additional fuel is studied numerically. Though the fusion energy which boron generates through the $\mathrm{p}-{ }^{11} \mathrm{~B}$ reaction is only $(1 \sim 5) \%$ of the whole, repetitive injection of solid boron pellets realizes stable burning of a low-temperature and high-density plasma. While the operating density is controlled by the deuterium fueling, a DB reactor can be operated in the thermally unstable regime by adjusting boron pellet injection based on the temperature measurements, from a low power to a high power output mode with reduced divertor heat flux.
\end{abstract}

(c) 2015 The Japan Society of Plasma Science and Nuclear Fusion Research

Keywords: advanced reactor, proton-boron reactor, catalyzed DD reactor, LHD

DOI: $10.1585 /$ pfr. 10.3405054

\section{Introduction}

In a DT reactor, the tritium self-production is necessary and large quantities of the neutron are produced. Although a $\mathrm{D}-{ }^{3} \mathrm{He}$ fusion reactor has less neutron and tritium production, ${ }^{3} \mathrm{He}$ resources are absent on the Earth. With respect to the quantities of fuel resources, a DD reactor is ideal one on the earth although substantial tritium and neutron are produced. However, in general it is more difficult to achieve ignition in a DD fusion than in $\mathrm{D}-{ }^{3} \mathrm{He}$ fusion because DD reactivity is smaller and the fusion product energy of DD reactions is smaller than that of $\mathrm{D}-{ }^{3} \mathrm{He}$ reactions by the factor of $18.34 \mathrm{MeV} / 4.86 \mathrm{MeV} \sim 4$. Ignition regime has two operating points in the thermally stable high temperature and the thermally unstable low temperature regimes. So far advanced fusion research has been done using the thermally stable high temperature regime [1]. In this high temperature regime, the synchrotron radiation loss is very large, and fueling is very difficult because ice pellet are immediately ionized at the plasma edge. Therefore, the lower temperature operation at the thermally unstable regime is attractive due to the possibility to lower the synchrotron radiation loss and to ease pellet injection.

So far the thermally unstable operating point has been proposed by controlling fueling such as deuterium ice pellet in FFHR reactor $[2,3]$. On the other hand, impurity

\footnotetext{
author's e-mail: omitarai@ktmail.tokai-u.jp

*) This article is based on the presentation at the 24th International Toki Conference (ITC24).

**) Deceased
}

injection proposed to stabilize the thermally unstable operation [4] does not so affect the operating density and the temperature mainly varies although ignition regime is narrowed slightly.

In this study, we propose a DD reactor operating in the thermally unstable low temperature regime controlled by solid ${ }^{11} \mathrm{~B}$ pellet injection, in which the deuterium is primary fuel and the boron is additional fuel (which is not impurity itself). In a deuterium-boron (DB) fusion reactor, (43 63)\% of the fusion energy (depending on the nuclear burning conditions) is released as charged particle energy $\left(=P_{\mathrm{c}}\right)$. We call this a DB reactor. The synchrotron radiation is smaller due to the lower temperature although it cannot be neglected. The high-energy protons generated by the D-D and $\mathrm{D}^{3}{ }^{3} \mathrm{He}$ fusion reactions produces the additional power from $\mathrm{p}^{11} \mathrm{~B}$ reaction. In a $\mathrm{DB}$ reactor, good confinement for high-energy particles (proton and ${ }^{3} \mathrm{He}$ ), which are produced by DD and $\mathrm{D}^{3} \mathrm{He}$ reactions, are necessary. The bremsstrahlung losses $\left(=P_{\text {brm }}\right)$ enhanced by the higher ion charge number $\left(Z_{\mathrm{B}}=5\right)$ of injected ${ }^{11} \mathrm{~B}$ may reduce the divertor heat load $\left(=P_{\text {div }}\right)$ and heat the blanket surface to convert to the electricity. To our knowledge, the thermally unstable low temperature operation is employed for the first time in the advanced fusion reactor study. To study such possible ignited operation, we use a helical reactor as an example [5] with parameters of $R_{0}=15 \mathrm{~m}, a_{\text {eff }}$ $=2.9 \mathrm{~m}, B_{\mathrm{ax}}=10 \mathrm{~T}, V_{\mathrm{lcfs}}=2000 \mathrm{~m}^{3}$, and $\gamma=1.2$, where $R_{0}, a_{\mathrm{eff}}, B_{\mathrm{ax}}, V_{\mathrm{lcfs}}$ and $\gamma$ are the major radius, the effective minor radius, the field intensity on the magnetic axis, the 
volume enclosed by the last closed flux surface (LCFS) and the helical coil pitch parameter, respectively.

In Sec. 2, we describe the zero dimensional model of a DB rector. In Sec. 3, we describe the numerical results for the burn control of DB reactors by boron pellet injection. We discuss the results in Sec. 4.

\section{Zero Dimensional Model of DB Re- actor}

Nuclear reactions of DB reactor fueled by deuterium and boron are as follows.

$$
\begin{aligned}
& \mathrm{D}+\mathrm{D} \rightarrow \mathrm{p}(3.03 \mathrm{MeV})+\mathrm{T}(1.01 \mathrm{MeV}), \\
& \mathrm{D}+\mathrm{D} \rightarrow n(2.45 \mathrm{MeV})+{ }^{3} \mathrm{He}(0.82 \mathrm{MeV}), \\
& \mathrm{D}+\mathrm{T} \rightarrow n(14.06 \mathrm{MeV})+{ }^{4} \mathrm{He}(3.52 \mathrm{MeV}), \\
& \mathrm{D}+{ }^{3} \mathrm{He} \rightarrow \mathrm{p}(14.67 \mathrm{MeV})+{ }^{4} \mathrm{He}(3.67 \mathrm{MeV}), \\
& \mathrm{p}+{ }^{11} \mathrm{~B} \rightarrow 3{ }^{4} \mathrm{He}(2.90 \mathrm{MeV})
\end{aligned}
$$

Then the zero dimensional model of a DB reactor is reduced to followings with external heating source per unit volume $\left(=P_{\text {aux }}\right)$.

$$
\begin{aligned}
\frac{\mathrm{d} W_{\mathrm{p}}}{\mathrm{d} t}= & P_{\mathrm{aux}}+P_{\mathrm{c}}-P_{\mathrm{cnd}}-P_{\mathrm{brm}}, \\
\frac{\mathrm{d} N_{\mathrm{D}}}{\mathrm{d} t}= & S_{\mathrm{D}}-N_{\mathrm{D}}\left[\langle\sigma v\rangle_{\mathrm{DT}} N_{\mathrm{T}}\right. \\
& +\left\{\langle\sigma v\rangle_{\mathrm{DDPT}}+\langle\sigma v\rangle_{\mathrm{DD}^{3} \mathrm{HeN}}\right\} N_{\mathrm{D}} \\
& \left.+\langle\sigma v\rangle_{\mathrm{D}^{3} \mathrm{He}} N^{3} \mathrm{He}\right], \\
\frac{\mathrm{d} N_{\mathrm{T}}}{\mathrm{d} t}= & \langle\sigma v\rangle_{\mathrm{DDPT}} \frac{N_{\mathrm{D}}^{2}}{2}-\langle\sigma v\rangle_{\mathrm{DT}} N_{\mathrm{D}} N_{\mathrm{T}}, \\
\frac{\mathrm{d} N_{3} \mathrm{He}}{\mathrm{d} t}= & \langle\sigma v\rangle_{\mathrm{DDn}{ }^{3} \mathrm{He}} \frac{N_{\mathrm{D}}^{2}}{2}-\langle\sigma v\rangle_{\mathrm{D}^{3} \mathrm{He}} N_{\mathrm{D}} N^{3} \mathrm{He}, \\
\frac{\mathrm{d} N_{\mathrm{p}}}{\mathrm{d} t}= & \langle\sigma v\rangle_{\mathrm{DDpT}} \frac{N_{\mathrm{D}}^{2}}{2}+\langle\sigma v\rangle_{\mathrm{D}^{3} \mathrm{He}} N_{\mathrm{D}} N^{{ }^{3} \mathrm{He}} \\
& -\langle\sigma v\rangle_{\mathrm{pB}} N_{\mathrm{p}} N_{\mathrm{B}}-\frac{N_{\mathrm{p}}}{\tau_{\mathrm{p}}}, \\
\frac{\mathrm{d} N_{\alpha}}{\mathrm{d} t}= & \langle\sigma v\rangle_{\mathrm{DT}} N_{\mathrm{D}} N_{\mathrm{T}}+\langle\sigma v\rangle_{\mathrm{D}^{3} \mathrm{He}} N_{\mathrm{D}} N^{3} \mathrm{He} \\
& +3\langle\sigma v\rangle_{\mathrm{pB}} N_{\mathrm{p}} N_{\mathrm{B}}-\frac{N_{\alpha}}{\tau_{\alpha}}, \\
\frac{\mathrm{d} N_{\mathrm{B}}}{\mathrm{d} t}= & S S_{\mathrm{B}}-\langle\sigma v\rangle_{\mathrm{pB}} N_{\mathrm{p}} N_{\mathrm{B}}-\frac{N_{\mathrm{B}}}{\tau_{\mathrm{B}}},
\end{aligned}
$$

where the plasma conduction loss is $P_{\mathrm{cnd}}=W_{\mathrm{p}} /\left(\tau_{\mathrm{iss} 95} h_{\mathrm{fct}}\right)$, the energy confinement time $\tau_{\text {iss } 95}$ is estimated by the ISS 95 scaling with the confinement improving factor $h_{\mathrm{fct}}$. In this study the confinement times $\left(\tau_{\mathrm{D}}, \tau_{\mathrm{T}}\right.$ and $\left.\tau_{{ }^{3} \mathrm{He}}\right)$ for $\mathrm{D}$, $\mathrm{T}$ and ${ }^{3} \mathrm{He}$ fueling particle are assumed to be large due to the high wall recycling, although it slightly overestimates DD ignition. Particle confinement times such as $\tau_{\mathrm{p}}, \tau_{\alpha}$ and $\tau_{\mathrm{B}}$ for a kind of ash particles are only considered for simplicity. $W_{\mathrm{p}}$ is the plasma thermal energy density per unit volume,

$$
\begin{aligned}
W_{\mathrm{p}}= & \frac{3}{2}\left[2 N_{\mathrm{T}}+2 N_{\mathrm{D}}+3 N_{3^{3} \mathrm{He}}+N_{\mathrm{p}}\left(T_{\mathrm{p}} / T_{\mathrm{e}}+1\right)\right. \\
& \left.+3 N_{\alpha}+6 N_{\mathrm{B}}\right] T_{\mathrm{e}} \times 1.602176462 \times 10^{-19}
\end{aligned}
$$

Here the electron density satisfying the charge neutral condition $N_{\mathrm{e}}=N_{\mathrm{T}}+N_{\mathrm{D}}+2 N^{3} \mathrm{He}+N_{\mathrm{p}}+5 N_{\mathrm{B}}$ is used, and other notations are the usual ones.

$$
\begin{aligned}
\tau_{\mathrm{iss} 95}= & \frac{0.2071513306 \times 10^{-8} N_{\mathrm{e}}^{0.51} B_{\mathrm{ax}}^{0.83} V_{\mathrm{lcfs}}^{1.105}}{\left(P_{\mathrm{c}}+P_{\mathrm{aux}}-P_{\mathrm{brm}}\right)^{0.59} R_{\mathrm{ax}}^{0.455}} \\
& \times\left(\frac{\iota}{2 \pi}\right)^{0.4} .
\end{aligned}
$$

The nuclear fusion reaction rate coefficients, $\langle\sigma \mathrm{v}\rangle_{\mathrm{DT}}$, $\langle\sigma \mathrm{v}\rangle_{\mathrm{DDpT}},\langle\sigma \mathrm{v}\rangle_{\mathrm{DDn}^{3} \mathrm{He}},\langle\sigma \mathrm{v}\rangle_{\mathrm{D}^{3} \mathrm{He}}$, are shown in the reference [6]. Since protons are produced by the reactions Eqs. $(1,4)$, they have high energy tail components, which contribute to $\mathrm{p}-{ }^{11} \mathrm{~B}$ reactions very much. Then, the nuclear reaction rate for $\langle\sigma \mathrm{v}\rangle_{\mathrm{pB}}$, is approximated by the reaction rate for high temperature protons. The functional form for $\langle\sigma \mathrm{v}\rangle_{\mathrm{pB}}$ is developed in the reference [7]. The functional forms for nuclear reaction rate are expressed as follows.

$$
\left.\begin{array}{l}
\langle\sigma v\rangle_{\mathrm{DT}} \\
\langle\sigma v\rangle_{\mathrm{D}^{3} \mathrm{He}}
\end{array}\right\}=\exp \left(-X_{1}-\frac{X_{2}}{T^{X_{5}}}+\frac{X_{3}}{T+X_{4}}\right),
$$

Coefficients are summarized in Table 1 and the nuclear fusion reaction rate coefficients $\langle\sigma v\rangle$ are shown in Fig. 1.

\section{Burn Control of a DB Reactor}

Solid boron pellets injection can control the quasisteady state burning of DB reactor in the thermally unstable regime. Burn control of the DB reactor by constant time interval $\left(=\tau_{\mathrm{d}}\right)$ injection of solid boron pellets are summarized as follows.

- Deuterium is fed constantly by gas puffing.

Table 1 Coefficients for the functional form for (1) DT, (2) $\mathrm{D}^{3} \mathrm{He}$, (3) DDpT, (4) $\mathrm{DDn}^{3} \mathrm{He}$, (5) $\mathrm{pB}\left(T_{\mathrm{p}}=T_{\mathrm{e}}\right)$, (6) $\mathrm{pB}\left(T_{\mathrm{p}}=4.5 T_{\mathrm{e}}\right)$, and $(7) \mathrm{pB}\left(T_{\mathrm{p}}=8 T_{\mathrm{e}}\right)$ reactions.

\begin{tabular}{|c|c|c|c|c|c|}
\hline & $X_{1}$ & $X_{2}$ & $X_{3}$ & $X_{4}$ & $X_{5}$ \\
\hline 1 & 48.958081 & 18.115508 & 895.149426 & 135.88863 & 0.366290 \\
\hline 2 & 47.610150 & 31.764777 & 2802.939515 & 374.18607 & 0.286712 \\
\hline 3 & 49.178972 & 15.326758 & -4168271.6 & 36677.969 & 0.365303 \\
\hline 4 & 48.993117 & 15.612510 & -430753.06 & 16887.313 & 0.363023 \\
\hline 5 & 48.573635 & 65.906939 & 81293510.05 & 103314.94 & 0.627586 \\
\hline 6 & 48.587615 & 69.464672 & 99871724.39 & 120056.14 & 0.631532 \\
\hline 7 & 48.589482 & 69.934518 & 102634755.0 & 122438.68 & 0.632008 \\
\hline
\end{tabular}




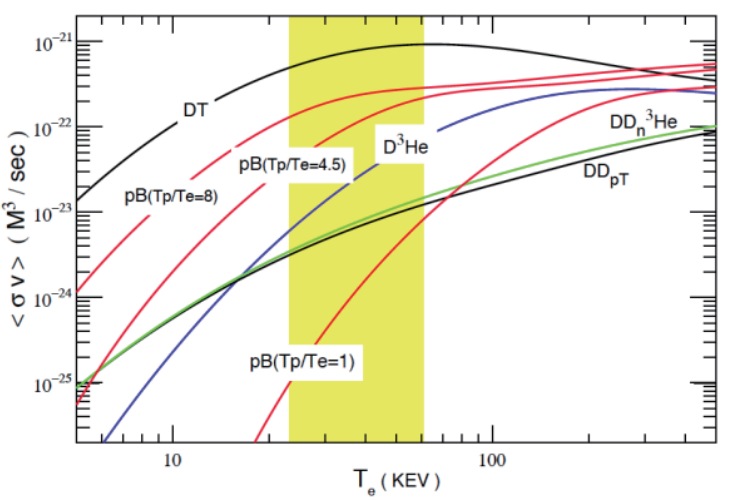

Fig. 1 Nuclear fusion reaction rate coefficients in a DB reactor. The horizontal and vertical axis represent plasma temperature and fusion reaction rate in DB reactors, respectively. For $\mathrm{p}-{ }^{11} \mathrm{~B}$ reactions, proton temperature is taken as a parameter.

- Plasma operation contours plot (POPCON plot) determines the boron pellet size. The atomic number of one boron pellet is given as follows.

$$
\begin{aligned}
& \begin{cases}0.4 \times 10^{17} \times V_{\text {lcfs }} \times \tau_{\mathrm{d}} & \left(T_{\mathrm{e}} \geq T_{\text {cntl }}\right), \\
0 & \left(T_{\mathrm{e}}<T_{\text {cntl }}\right),\end{cases} \\
& T_{\text {cntl }}=(23 \sim 50) \mathrm{keV} .
\end{aligned}
$$

- A kind of ash particles such as boron, proton and helium 4 are diffused away.

A POPCON plot is obtained from steady state solutions of Eqs. (6-12), where a contour plot of $P_{\mathrm{AUX}}$ is drawn in $\left(T_{\mathrm{e}}, N_{\mathrm{e}}\right)$ plane for the given fuel density ratio $N_{\mathrm{B}} / N_{\mathrm{D}}$. The POPCON plot can overwrite the contour plot of the fuel sources $\left(S_{\mathrm{D}}, S_{\mathrm{B}}\right)$, and the plasma conduction loss $P_{\text {cnd }}$. The divertor heat flux is estimated by $P_{\mathrm{div}}=P_{\mathrm{CND}} /(2 \pi R \Delta 4)$ for the assumed divertor wet width of $\Delta=0.1 \mathrm{~m}$ at a steady state burning plasma. If the temperature $T_{\mathrm{e}}$ is higher than $T_{\text {cntl }}\left(T_{\mathrm{e}}>T_{\text {cntl. }}\right)$, slightly larger size pellets of ${ }^{11} \mathrm{~B}$ are injected, and then the operating point moves to the lower temperature side. When the temperature $T_{\mathrm{e}}$ is lower than $T_{\text {cntl }},{ }^{11} \mathrm{~B}$ pellet injection is stopped, then the temperature is increased. Thus, the operation point is stabilized in the thermally unstable regime.

The operating path on the POPCON plot is shown in Fig. 2 for the confinement improving factor $h_{\mathrm{fct}}=8$. To demonstrate the existence of DD ignition regime, rather large value of the confinement improving factor $h_{\mathrm{fct}}=8$ has been employed as the first trial. In Fig. 2 is shown the stability of operating point near the thermally unstable ignition boundary. Here the fusion power output is low and the divertor heat flux is also minimized. The green curve shows the time trace of the burning points starting from $T_{\mathrm{e}}=10 \mathrm{keV}$ with $\left(S_{\mathrm{D}}, S_{\mathrm{B}}\right)=(20.5,75.0) \times 10^{17} \mathrm{~m}^{-3} / \mathrm{sec}$, $\left(N_{\mathrm{D}}, N_{\mathrm{B}}\right)=(4.5,0) \times 10^{19} \mathrm{~m}^{-3}$. The external heating power of $P_{\mathrm{AUX}}=70 \mathrm{MW}$ is applied for $50 \mathrm{sec}$, and switched off. It is clearly shown the operating point is stabilized by boron pellet injections at the set point of the temperature at $T_{\text {cntl }}$

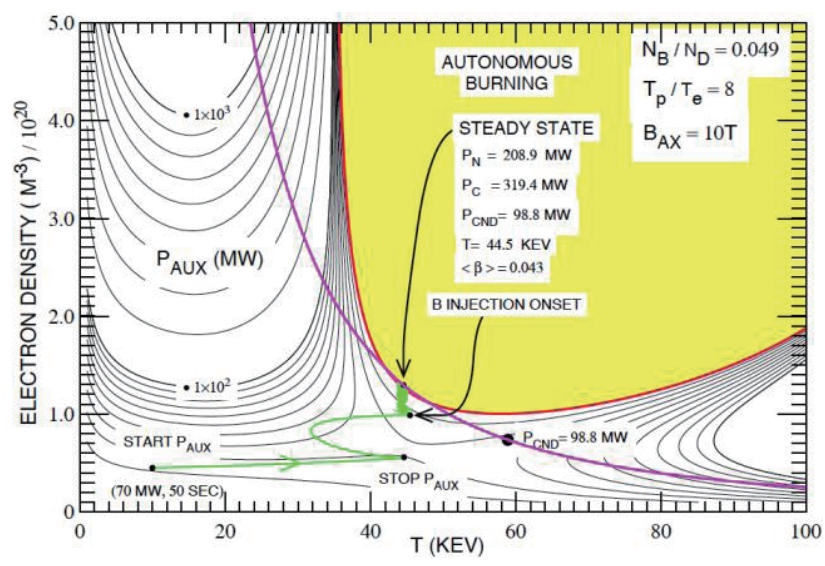

Fig. 2 The operating point near the thermally unstable ignition boundary on POPCON controlled by the boron pellet injection at $T_{\text {cntl }}=44.55 \mathrm{keV}$ and $N_{\mathrm{B}} / N_{\mathrm{D}}=0.049$. The fusion output is $P_{\mathrm{F}}\left(=P_{\mathrm{c}}+P_{\mathrm{N}}\right)=528.3 \mathrm{MW}$ and the plasma conduction loss is $P_{\mathrm{CND}}=98.8 \mathrm{MW}$ at the steady state burning point.

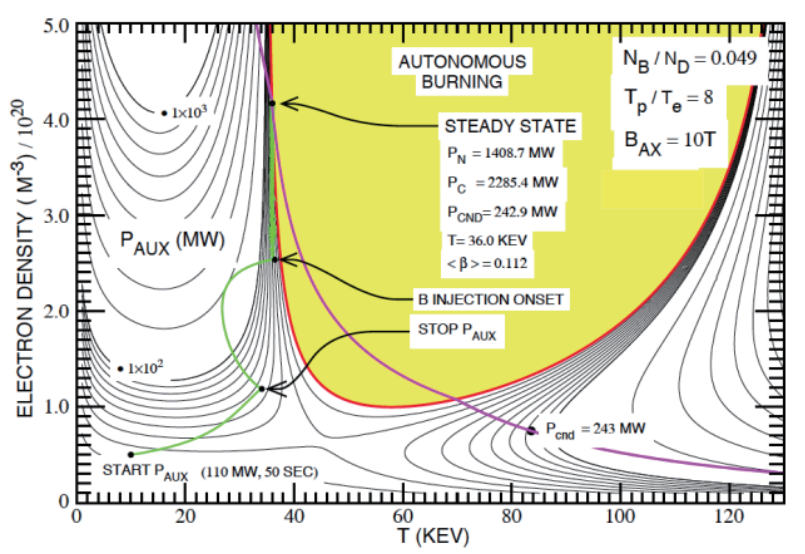

Fig. 3 The time trace (shown by a green curve) of the operating point on POPCON for a high power fusion output mode $\left(P_{\mathrm{F}}=3.69 \mathrm{GW}\right)$ with reduced divertor heat flux of $P_{\text {div }}=$ $6.4 \mathrm{MW} / \mathrm{m}^{2}\left(P_{\mathrm{CND}}=242.9 \mathrm{MW}\right) . T_{\mathrm{cntl}}=36 \mathrm{keV}$.

$=44.55 \mathrm{keV}$ on the thermally unstable ignition boundary.

By increasing the deuterium fueling, the operating point moved up to the final operating point with the high power fusion output mode and the reduced divertor heat flux as shown in Fig. 3 and Fig. 4. The operating point accesses the thermally unstable steady state point from the starting point with $T_{\mathrm{e}}=10 \mathrm{keV}$, and $\left(S_{\mathrm{D}}, S_{\mathrm{B}}\right)=(14.0$, $21.0) \times 10^{17} \mathrm{~m}^{-3} / \mathrm{sec},\left(N_{\mathrm{D}}, N_{\mathrm{B}}\right)=(5,0) \times 10^{19} \mathrm{~m}^{-3}$. The external heating power of $P_{\mathrm{AUX}}=110 \mathrm{MW}$ is applied for $50 \mathrm{sec}$. After over-passing the peak line of the heating power on POPCON (STOP $P_{\mathrm{AUX}}$ in Fig. 3), the external heating power is switched off. Then the operating point moves up to the ignition regime by deuterium fueling and the fusion product heating such as $\mathrm{p},{ }^{3} \mathrm{He}, \mathrm{T}$, and alpha heating without external heating power. The temperature once decreases due to switching off the external heating 


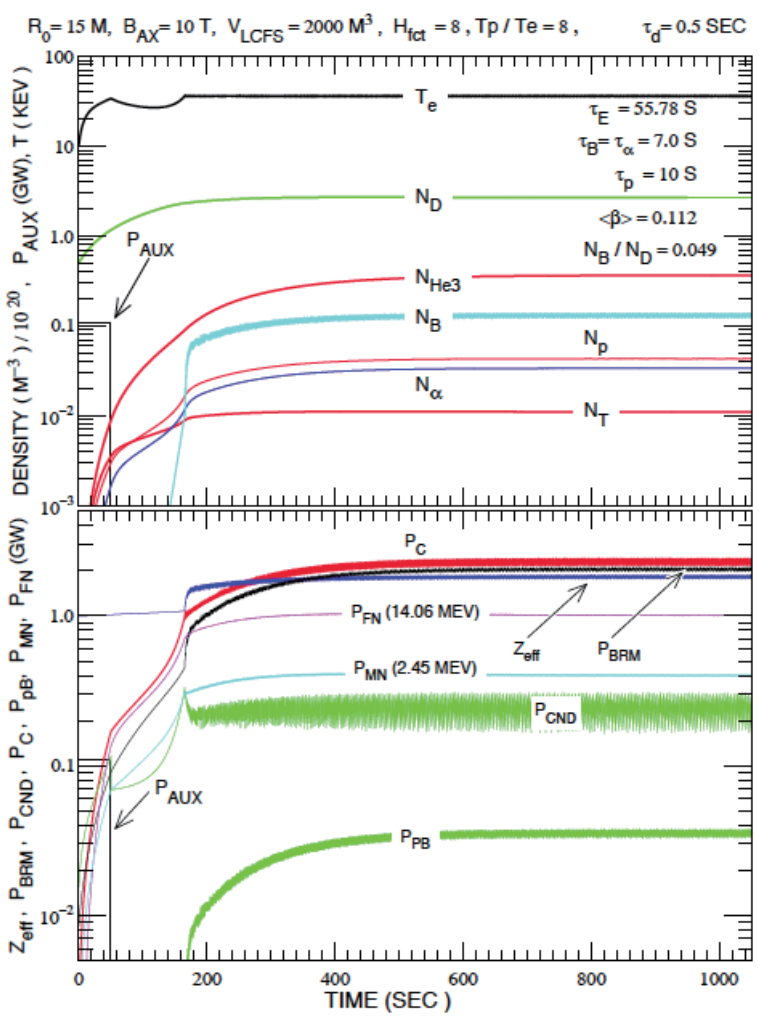

Fig. 4 Temporal evolution of the plasma parameters to the high power fusion output mode with reduced divertor heat flux $\left(P_{\mathrm{F}}=3.69 \mathrm{GW}, P_{\mathrm{CND}}=242.9 \mathrm{MW}\right)$ shown in Fig. 3 . Shorter ash particle confinement times $\left(\tau_{\mathrm{B}}=\tau_{\alpha}=7 \mathrm{~s}\right.$ and $\tau_{\mathrm{p}}=10 \mathrm{~s}$ ) are essential for DD ignition in this study.

power and fueling, and then increases again by the fusion product heating. The operating point finally approaches the setting value of $T_{\mathrm{cntl}}=36 \mathrm{keV}$, where boron pellets start to be injected. It is then seen that the operating point straightly proceeds to the higher-density steady state operating point along the $T_{\text {cntl }}=36 \mathrm{keV}$ line by deuterium fueling $\left(S_{\mathrm{D}}\right)$ and boron pellet injections $\left(S_{\mathrm{B}}\right)$. By comparing the Fig. 2 and 3, it is seen that the operating point can be adjusted by the set value of the temperature $T_{\text {cntl }}$.

The temporal evolutions of the various parameters are shown in Fig. 4. It is clearly seen that the ignition is achieved after the external heating power is switched off. The core plasma is kept steadily at thermally unstable point $\left(T_{\mathrm{e}}=36.0 \mathrm{keV}, N_{\mathrm{e}}=4.16 \times 10^{20} \mathrm{~m}^{-3}\right)$ by the repetitive solid boron pellets injection $\left(\tau_{\mathrm{d}}=0.5 \mathrm{sec}\right)$. Average fusion output from $\mathrm{p}^{11} \mathrm{~B}$ reaction is only $P_{\mathrm{pB}}=35.2 \mathrm{MW}$, and the bremsstrahlung power is $P_{\mathrm{BRM}} \sim 2 \mathrm{GW}$ and the neutron powers are $\sim 1 \mathrm{GW}$ and $\sim 0.4 \mathrm{GW}$ by $14.06 \mathrm{MeV}$ and $2.45 \mathrm{MeV}$, respectively. This large bremsstrahlung power could be converted to the heat and electricity although conversion system should be studied more.

Figure 5 shows the changes in the steady state of the core plasma as a function of a plasma temperature (from thermally unstable regime to thermally stable regime).

In high-temperature and low-density core plasma, the divertor heat flux $P_{\text {div }}$ becomes large, because the

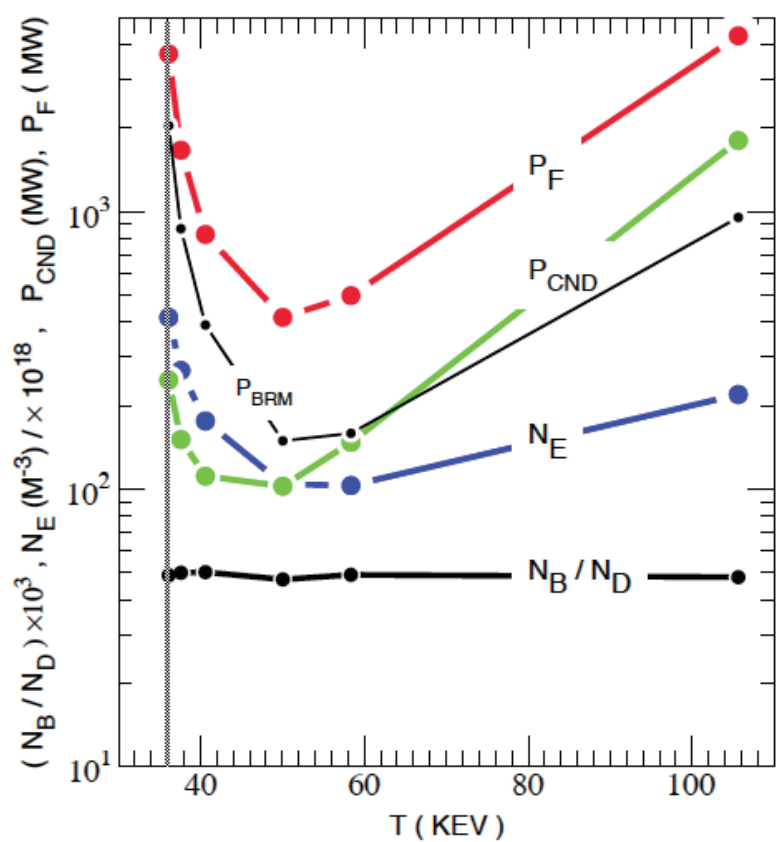

Fig. 5 Temperature dependency of the various powers for $N_{\mathrm{B}} / N_{\mathrm{D}}=0.05$. At $T_{\mathrm{e}}=36 \mathrm{keV}$ (vertical gray line), the bremsstrahlung power $P_{\mathrm{BRM}}$ approaches the fusion power $P_{\mathrm{F}}$, and conduction power $P_{\mathrm{CND}}$ does not increase so much, then the divertor heat flux is reduced but not the minimum.

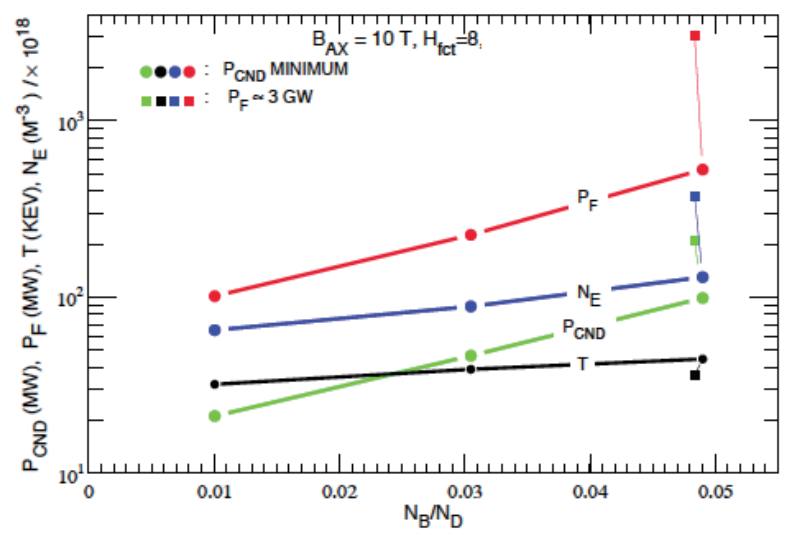

Fig. $6 N_{\mathrm{B}} / N_{\mathrm{D}}$ dependence of the minimum divertor heat flux mode shown in Fig. 5 .

bremsstrahlung loss is much smaller than the charged particle energy $\left(P_{\mathrm{BRM}} \ll P_{\mathrm{c}}\right)$. On the other hand, in the low-temperature and high-density core plasma, the divertor heat flux can be reduced very much because the bremsstrahlung loss $P_{\mathrm{BRM}}$ is close to the same level of the charged particle energy $P_{\mathrm{c}}$. Furthermore, Fig. 5 shows the existence of the plasma temperature to minimize a divertor heat flux derived from $P_{\mathrm{CND}}$.

The $N_{\mathrm{B}} / N_{\mathrm{D}}$ dependence of the minimum divertor heat flux mode is summarized in Fig. 6. Autonomous burning area decreases with increasing of the density ratio $N_{\mathrm{B}} / N_{\mathrm{D}}$. It is recognized that core plasma control becomes difficult 
when the density ratio, $N_{\mathrm{B}} / N_{\mathrm{D}}$, is too small. For $N_{\mathrm{B}} / N_{\mathrm{D}}=$ 0.0483 , the burning point with $P_{\mathrm{F}}=3.02 \mathrm{GW}$ (the high power fusion output and the reduced divertor heat flux mode) is shown by square marks for comparison.

\section{Discussions}

In the present paper, we showed the possibility of quasi-steady state operation of low-temperature and highdensity operation in an advanced DD fusion reactor (DB reactor) using controlled solid boron pellets injection. Repetitive injection of solid boron pellets stabilizes the operating point in the thermally unstable regime with a low-temperature and high-density plasma. Broad operating regimes $\left(100 \mathrm{MW} \leq P_{\mathrm{F}} \leq 3.69 \mathrm{GW}, 21 \mathrm{MW} \leq P_{\mathrm{CND}} \leq\right.$ $243 \mathrm{MW}$ ) can be realized by the regulation of boron pellet size injection and deuterium fueling.

Large size boron pellets are advantageous for injection into the deep core plasma. Equation for the boron density (12) gives the same results, when the product of boron particle life time and source term of boron, $\tau_{\mathrm{B}} \times S_{\mathrm{B}}$, has the same values. Then, if the boron particle life time $\tau_{\mathrm{B}}$ become short by the impurity hole phenomena [8], large size boron pellets become possible to use.

In the present paper, the high-energy tail component of protons are approximated by high temperature $T_{\mathrm{p}}$. The calculation of reaction rate using the distribution function of proton and ${ }^{3} \mathrm{He}$ obtained by a Boltzmann-Fokker-Planck equation is a future subject.

Although a helical reactor with the bold assumptions was used to show the existence of the DD ignition regime in this study, further studies would be pursued using a spherical tokamak (ST). Tentative parameters are the major radius $R \sim 6.7 \mathrm{~m}$, minor radius $a \sim 4.2 \mathrm{~m}$, the toroidal field $B=4.0$ T to reduce the synchrotron radiation loss with the higher beta value, and the plasma current of $\sim 100 \mathrm{MA}$ to reduce the confinement improving factor to 2.5 over IPB98y 2 scaling. The peaked temperature and density profiles would be used, lowering the required confinement improving factor. As ${ }^{11} \mathrm{~B}$ (the melting point $2075^{\circ} \mathrm{C}$ ) might be mechanically stronger than the ice pellet, the injector speed can be increased. Estimation of evaporation of solid pellet of ${ }^{11} \mathrm{~B}$ might be crucially important in this DB reactor study. In summary although bold assumptions have been employed, this paper stimulates the further study of the thermally stable and unstable operation in an advanced fuel fusion reactor, and also shows that the shorter ash particle confinement time is important to achieve DD ignition.

This work was performed with the support and under the auspices of the NIFS Collaborative Research Programs NIFS13KNST062.

[1] O. Mitarai "A D- ${ }^{3} \mathrm{He}$ spherical tokamak reactor with the plasma current ramp-up by vertical field" in Nuclear Reactors, Nuclear Fusion and Fusion Engineering (Nova Science Publishers, Inc., 2009), Edited by A. Aasen and P. Olsson.

[2] O. Mitarai et al., Plasma Fusion Res. 2, 021 (2007).

[3] O. Mitarai et al., Plasma Fusion Res. 5, S1001 (2010).

[4] J. Mandrekas et al., Fusion Technol. 19, 57 (1991).

[5] T. Watanabe et al., Plasma Fusion Res. 9, 3403089 (2014).

[6] T. Watanabe et al., J. Plasma Fusion Res. 74, 850 (1998) [in Japanese].

[7] H. Hojo, "Theoretical Study on Proton-Boron Fusion Reactor with LHD-Type Magnetic Field Configuration", https://kaken.nii.ac.jp/d/p/15540480/2004/3/ja.ja.html (2004).

[8] Yoshinuma et al., Nucl. Fusion 49, 062002 (2009). 